\title{
Jump conditions for pressure anisotropy and comparison with the Earth's bow shock
}

\author{
D. F. Vogl ${ }^{1,2}$, H. K. Biernat ${ }^{1,2,3}$, N. V. Erkaev ${ }^{4}$, C. J. Farrugia ${ }^{5}$, and S. Mühlbachler ${ }^{1,2}$ \\ ${ }^{1}$ Space Research Institute, Austrian Academy of Sciences, Graz, Austria \\ 2 also at: Institute for Geophysics, Astrophysics, and Meteorology, University of Graz, Austria \\ ${ }^{3}$ also at: Institute for Theoretical Physics, University of Graz, Austria \\ ${ }^{4}$ Institute of Computational Modelling, Russian Academy of Sciences, Krasnoyarsk, Russia \\ ${ }^{5}$ Institute for the Study of Earth, Oceans, and Space, University of New Hampshire, Durham, USA
}

Received: 6 July 2000 - Revised: 4 October 2000 - Accepted: 11 October 2000

\begin{abstract}
Taking into account the pressure anisotropy in the solar wind, we study the magnetic field and plasma parameters downstream of a fast shock, as functions of upstream parameters and downstream pressure anisotropy. In our theoretical approach, we model two cases: a) the perpendicular shock and b) the oblique shock. We use two threshold conditions of plasma instabilities as additional equations to bound the range of pressure anisotropy. The criterion of the mirror instability is used for pressure anisotropy $p_{\perp} / p_{\|}>1$. Analogously, the criterion of the fire-hose instability is taken into account for pressure anisotropy $p_{\perp} / p_{\|}<1$. We found that the variations of the parallel pressure, the parallel temperature, and the tangential component of the velocity are most sensitive to the pressure anisotropy downstream of the shock. Finally, we compare our theory with plasma and magnetic field parameters measured by the WIND spacecraft.
\end{abstract}

\section{Introduction}

For a collisionless magnetoplasma, Chew, Goldberger, and Low (1956) derived the quasi-magnetohydrodynamic equations with an anisotropic pressure tensor. This pressure tensor is characterized by two scalar pressures, i.e.

$P_{i k}=p_{\perp} \delta_{i k}+\left(p_{\|}-p_{\perp}\right) B_{i} B_{k} / B^{2}$,

where $p_{\perp}$ and $p_{\|}$are the pressures perpendicular and parallel with respect to the magnetic field, respectively. For the strong magnetic field approximation, the two pressures are related to the plasma density and the magnetic field strength by two adiabatic equations,

$\frac{d}{d t}\left(\frac{p_{\|} B^{2}}{\rho^{3}}\right)=0, \quad \frac{d}{d t}\left(\frac{p_{\perp}}{\rho B}\right)=0$.

Correspondence to: $\mathrm{H}$. K. Biernat

(helfried.biernat@kfunigraz.ac.at)
This approach is known as the double adiabatic theory.

The general jump conditions for discontinuities in a collisionless anisotropic magnetoplasma in the Chew-Goldberger-Low approximation were derived by Abraham-Shrauner (1967). Lynn (1967) made a qualitative analysis of the jump conditions for the change of plasma parameters between two stationary, uniform plasma regions and specified them as contact, tangential, rotational discontinuities, and compressible shocks. For the latter, the coplanarity theorem was taken into account. Neubauer (1970) obtained solutions of the jump relations for shocks moving into a collisionless anisotropic magnetized plasma under the assumption of isotropic conditions downstream of the shock front. Furthermore, Hudson (1970) discussed the types of discontinuities in a magnetohydrodynamic fluid with anisotropic plasma pressure and gave rules based on their identification in the solar wind.

Evidence for pressure anisotropy in the solar wind comes from spacecraft measurements. The Ames Research Center (ARC) plasma probe on Pioneer 6 showed that the thermal anisotropy, in general, has $T_{\|}>T_{\perp}$. A possible explanation on this special feature of the solar wind is discussed by Scarf et al. (1967), taking into account the conservation of the first adiabatic invariant. The opposite case, $T_{\|}<T_{\perp}$, is related to strong local ion heating by macroscale compressions or plasma instabilities (Bame et al., 1975).

Experimental data aspects were further included in the work by Chao et al. (1995). They analyzed the Rankine Hugoniot equations for the so-called quasi-perpendicular shock for low plasma beta and isotropic conditions $\left(T_{\perp}=\right.$ $T_{\|}$) upstream of the shock. Using AMPTE/IRM spacecraft data, they obtained the pressure anisotropy rate downstream of the shock, as a function of the plasma betas and the ratio of the magnetic field strengths across the shock. However, their approach is not suitable for predicting magnetosheath parameters as functions of the upstream solar wind param- 
eters, since their solutions are only applicable for concrete local data analysis at the shock.

To solve the jump equations for anisotropic plasma conditions upstream and downstream of the shock, one has to use an additional equation, since the set of equations is underdetermined. Therefore, experimental studies of the thermal behaviour of the magnetosheath, the region between the bow shock and the magnetopause, might be taken into account. Using data from the AMPTE/IRM spacecraft, Hill et al. (1995) have shown that the double adiabatic equations do not hold in the magnetosheath. Moreover, the thermal behaviour of the magnetosheath is studied by Phan et al. (1996) using WIND spacecraft data. They report that most parts of the magnetosheath are marginally mirror unstable.

Erkaev et al. (2000) solved the set of jump equations for perpendicular and oblique shocks for arbitrary pressure anisotropy downstream of the shock. In their analysis, magnetosheath parameters, as functions of upstream solar wind parameters, are obtained in a wide range of Alfvén Mach numbers ( $\left.3 \leq M_{A} \leq 10\right)$ without any restrictions on the Rankine Hugoniot equations. It is important to note that their calculations are applicable for a wide range of plasma betas, various pressure anisotropy rates, and different shock geometries upstream of the fast shock wave. Furthermore, Erkaev et al. (2000) used the criteria of the fire-hose and mirror instability as additional equations to determine the pressure anisotropy downstream of the shock. These two threshold conditions give some additional restrictions to the behaviour of the plasma upstream and downstream of the shock wave and therefore, the anisotropy rate cannot be outside of the interval determined by the threshold of these two plasma instabilities. Since the Rankine Hugoniot equations can only be applied to regions close to the shock wave, the use of these plasma instabilities, which are identified by spacecraft missions (e.g. Hill et al., 1995; Phan et al., 1996), gives some boundaries for the pressure anisotropy for either side of the shock. Different pressure anisotropy rates in the solar wind were examined by Biernat et al. (2000), Kiendl et al. (2000) and Vogl et al. (2000).

This paper is organized as follows: in the next section, we discuss the basic equations and specify the input parameters. What follows is the study of the variations of the plasma parameters and the magnetic field strength from upstream to downstream for the perpendicular and the oblique shock following the technique performed by Erkaev et al. (2000). In Sect. 4, we use a data example from the WIND spacecraft to determine the input parameters, solve the set of equations, and compare our theoretical results with the measurements.

\section{Basic equations}

The general jump conditions for a discontinuity in an anisotropic magnetoplasma are given by (Hudson, 1970)

$$
\llbracket \rho v_{n} \rrbracket=0
$$

$$
\begin{aligned}
& \llbracket v_{n} B_{t}-v_{t} B_{n} \rrbracket=0, \\
& \llbracket p_{\perp}+\left(p_{\|}-p_{\perp}\right) \frac{B_{n}^{2}}{B^{2}}+\frac{B_{t}^{2}}{8 \pi}+\rho v_{n}^{2} \rrbracket=0, \\
& \llbracket \frac{B_{n} B_{t}}{4 \pi}\left(\frac{4 \pi\left(p_{\|}-p_{\perp}\right)}{B^{2}}-1\right)+\rho v_{n} v_{t} \rrbracket=0, \\
& \llbracket \rho v_{n}\left(\frac{\mathcal{E}}{\rho}+\frac{v^{2}}{2}+\frac{p_{\perp}}{\rho}+\frac{B_{t}^{2}}{4 \pi \rho}\right)+\frac{B_{n}^{2} v_{n}}{B^{2}}\left(p_{\|}-p_{\perp}\right) \\
& \quad-\frac{\left(B_{t} \cdot v_{t}\right) B_{n}}{4 \pi}\left(1-\frac{4 \pi\left(p_{\|}-p_{\perp}\right)}{B^{2}}\right) \rrbracket=0, \\
& \llbracket B_{n} \rrbracket=0,
\end{aligned}
$$

where $\rho$ is the mass density, $v$ and $B$ are the velocity and magnetic field strength. Subscripts $t$ and $n$ indicate tangential and normal components with respect to the discontinuity. Quantities $p_{\perp}$ and $p_{\|}$are the elements of the plasma pressure tensor perpendicular and parallel with respect to the magnetic field. Quantity $\mathcal{E}$ is the internal energy, $\mathcal{E}=p_{\perp}+p_{\|} / 2$, and $\llbracket Q \rrbracket=Q_{2}-Q_{1}$, where subscripts 1 and 2 signify the quantity $Q$ upstream and downstream of the discontinuity.

As mentioned above, we use the mirror instability criterion as an additional relation to determine the pressure anisotropy downstream of the shock (Hasegawa, 1975),

$1+\sum_{\text {species }} \beta_{\perp}\left(1-\frac{\beta_{\perp}}{\beta_{\|}}\right)<0$.

In our calculations, we deal with two dimensionless parameters, $A_{S}$ and $A_{M}$, which are determined for upstream conditions as $A_{S}=p_{\perp 1} /\left(\rho_{1} v_{1}^{2}\right)$ and $A_{M}=1 / M_{A}^{2}$, where $M_{A}$ is the Alfvén Mach number. Furthermore, we introduce a coordinate system to determine the components of the velocity and the magnetic field strength upstream of the shock with respect to the discontinuity (see Fig. 1). For shocks, the tangential components of the electric and magnetic fields are coplanar. Thus, the components of the magnetic field upstream of the shock are given as $B_{n 1}=B_{1} \cos \gamma$ and $B_{t 1}=B_{1} \sin \gamma$, where $\gamma$ is the angle between the magnetic field vector and the vector normal to the discontinuity. Similarly, the components of the bulk velocity upstream of the shock are chosen as $v_{n 1}=v_{1} \cos \alpha$ and $v_{t 1}=v_{1} \sin \alpha$, where $\alpha$ the angle between the bulk velocity and the normal component of the velocity. Furthermore, we introduce a parameter $\lambda=p_{\perp} / p_{\|}$ which determines the pressure anisotropy. Using this parameter, we are able to express the parallel pressure upstream of the shock as $p_{\| 1}=p_{\perp 1} / \lambda_{1}$. From the conservation of mass, we define $y=\rho_{1} / \rho_{2}$ and $B_{n 1}=B_{n 2}$ is taken into account.

\section{Results}

\subsection{Solution for the perpendicular shock}

First, we study the more simple particular case of the socalled perpendicular shock, where $B_{n}=0$. Thus, Eqs. (4) to 


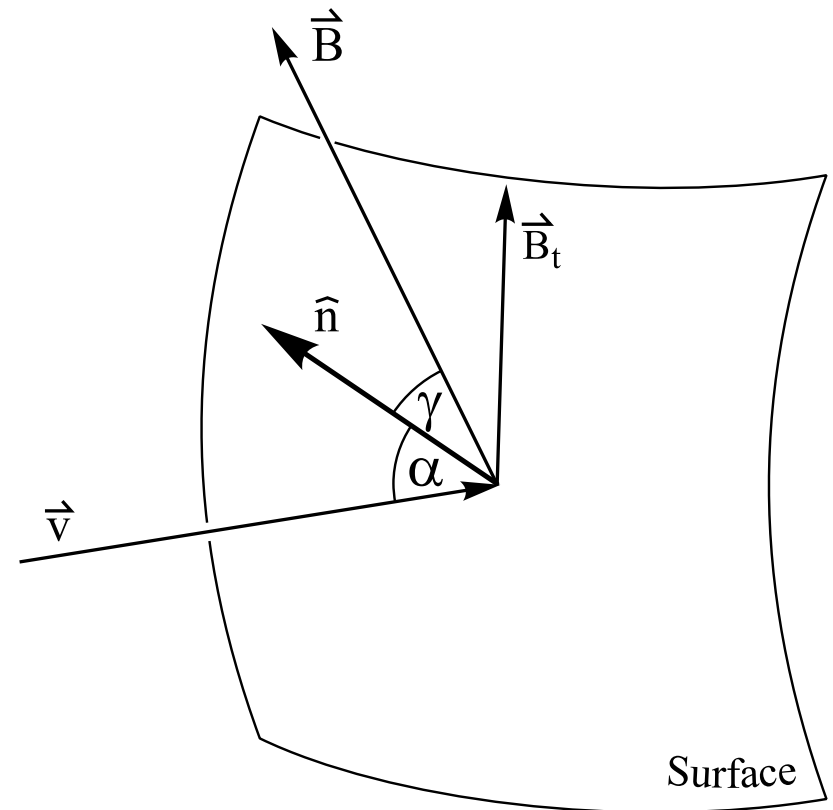

Fig. 1. Sketch for the geometric situation of the problem.

(7) reduce to

$$
\begin{aligned}
& \llbracket \rho v_{n} \rrbracket=0, \\
& \llbracket v_{n} B_{t} \rrbracket=0 \\
& \llbracket\left[p_{\perp}+\frac{B_{t}^{2}}{8 \pi}+\rho v_{n}^{2} \rrbracket=0,\right. \\
& \llbracket \rho v_{n} v_{t} \rrbracket=0, \\
& \llbracket \rho v_{n}\left(\frac{\mathcal{E}}{\rho}+\frac{v_{n}^{2}}{2}+\frac{v_{t}^{2}}{2}+\frac{p_{\perp}}{\rho}+\frac{B_{t}^{2}}{4 \pi \rho}\right) \rrbracket=0 .
\end{aligned}
$$

The quantities downstream of the discontinuity are

$B_{t 2}=x B_{t 1}$,

$v_{t 2}=v_{t 1}$,

$p_{\perp 2}=p_{\perp 1}+\frac{B_{t 1}^{2}}{8 \pi}\left(1-x^{2}\right)+\rho_{1} v_{n 1}^{2}\left(1-\frac{1}{x}\right)$.

Substituting Eqs. (15)-(17) into the energy Eq. (14), leads to

$$
\begin{aligned}
& 2 \lambda_{1}\left(3 \lambda_{2}+1\right) y^{3}-\lambda_{1}\left(4 \lambda_{2}+1\right)\left(2 A_{S}+A_{M}+2\right) y^{2} \\
& \quad+\lambda_{2}\left[2 \lambda_{1}\left(4 A_{S}+1+2 A_{M}\right)+2 A_{S}\right] y+A_{M} \lambda_{1}=0,
\end{aligned}
$$

where $y=1 / x$.

Figure 2 shows, from top to bottom, the variations of the plasma density, the velocity, the pressures and the temperatures perpendicular and parallel to the magnetic field downstream of the shock as functions of the anisotropy parameter, $\lambda_{2}$, for different Alfvén Mach numbers varying from 2 to 10 . For all panels $A_{S}=0.01$ and $\lambda_{1}=0.25$ is used.

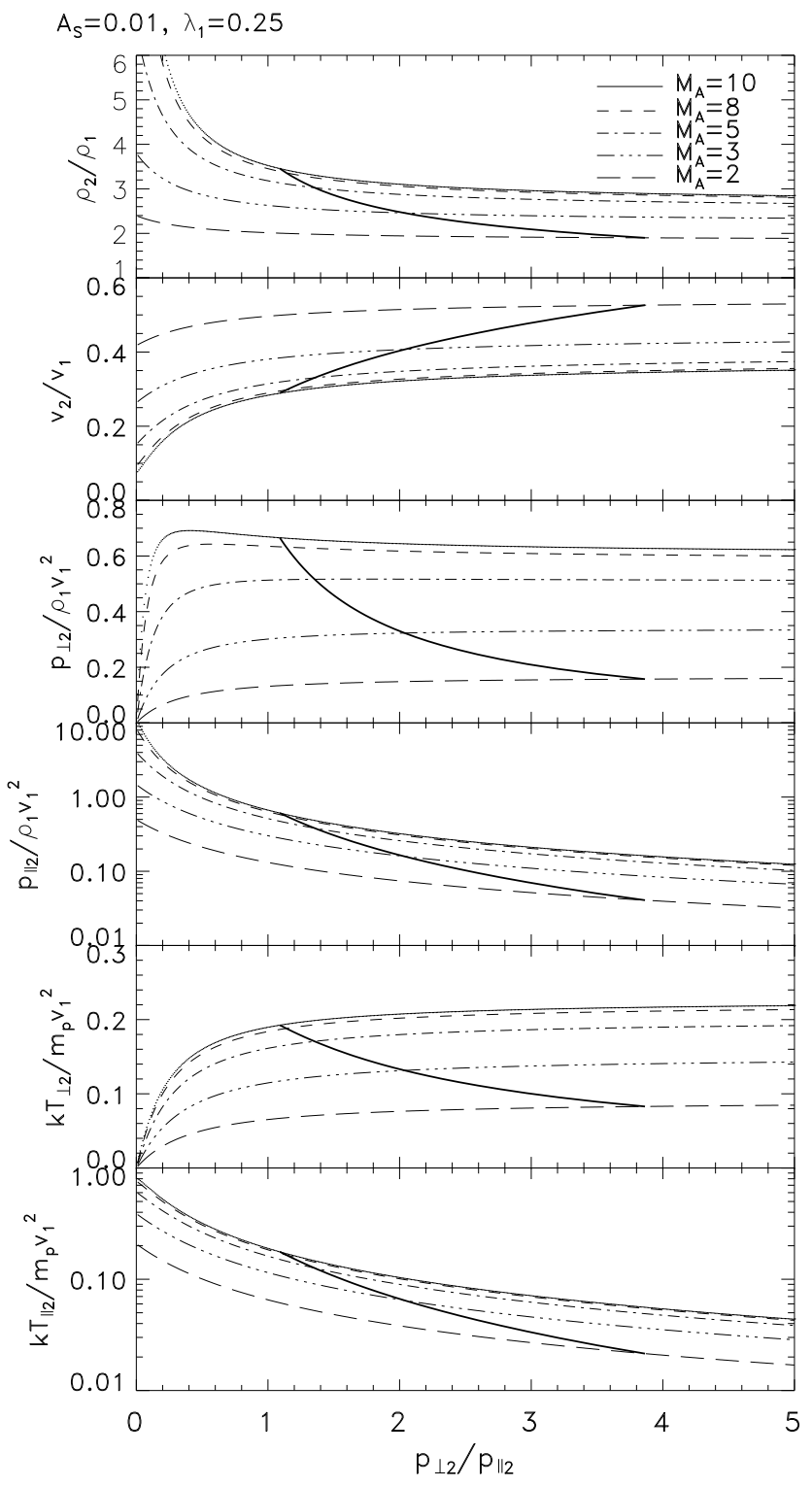

Fig. 2. Plasma parameters as functions of the anisotropy rate downstream of the perpendicular shock for $A_{S}=0.01$ and $\lambda_{1}=0.25$.

The thick line appearing in each panel represents the mirror criterion which divides all panels into stable (left side) and unstable (right side) regions. All curves are monotonic functions of the anisotropy rate, $\lambda_{2}$, and the maximum of the anisotropy parameter, bounded by the mirror instability, increases substantially as $M_{A}$ decreases. As seen from the figure, the changes of all parameters from upstream to downstream are strongly influenced by the Alfvén Mach number. High Alfvén Mach numbers correspond to stronger changes in the plasma parameters than low Alfvén Mach numbers. Figure 3 shows the same quantities as seen in Fig. 2, but for $A_{S}=0.04$. In Fig. 4, we show the plasma parameters as functions of the Alfvén Mach number for different values of $A_{S}, A_{S}=0.01$ and $A_{S}=0.04$. 


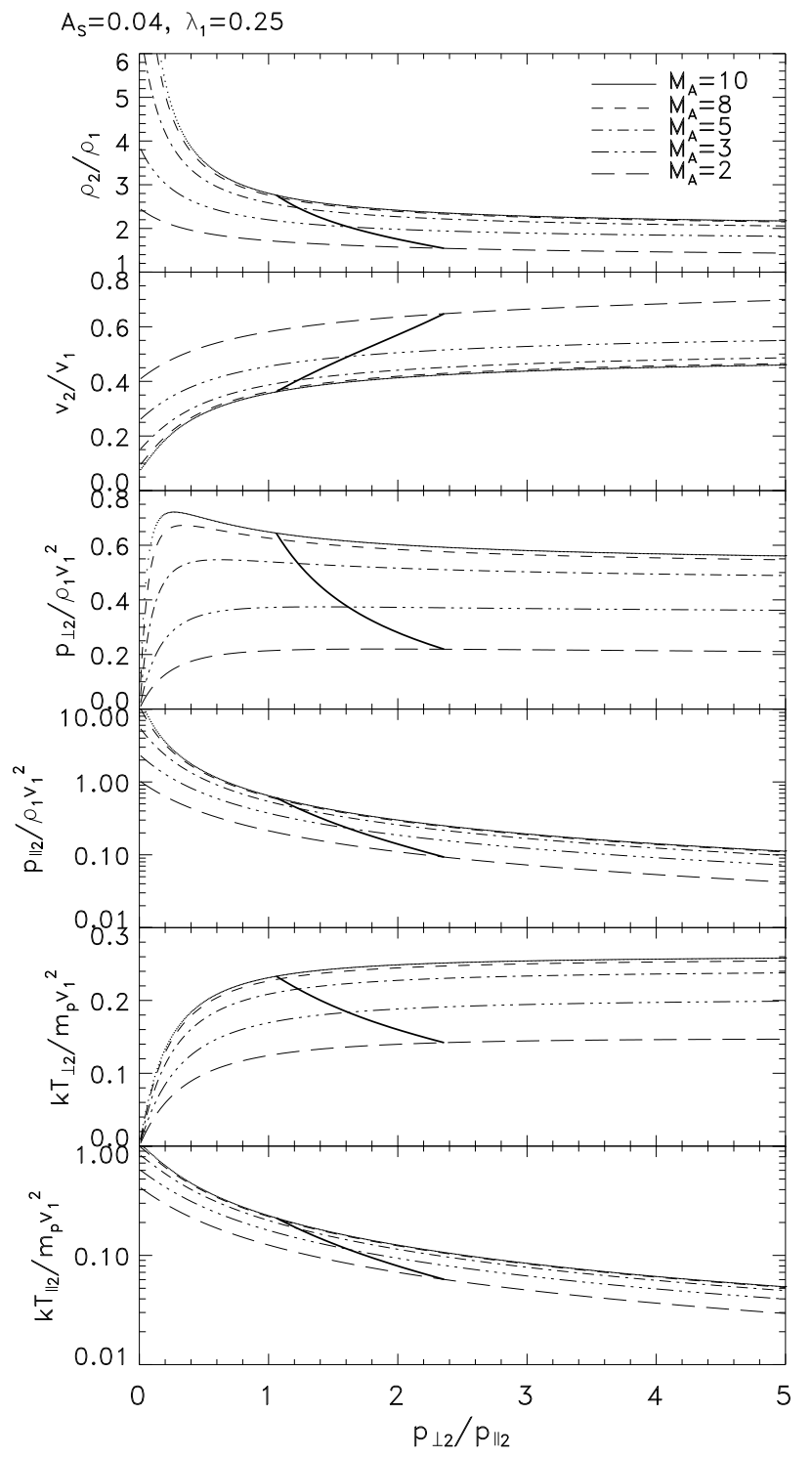

Fig. 3. Plasma parameters as functions of the anisotropy rate downstream of the perpendicular shock for $A_{S}=0.04$ and $\lambda_{1}=0.25$.

\subsection{Solution for the oblique shock}

In this section, we derive the jump equation for the case of an oblique shock. Solving the set of Eqs. (4-8), we introduce the parameter, $\varepsilon$,

$\varepsilon=1-\frac{4 \pi\left(p_{\|}-p_{\perp}\right)}{B_{2}^{2}}$

and obtain two equations which have to be solved simultaneously (Erkaev et al., 2000),

$\mathcal{A}_{4} y^{4}+\mathcal{A}_{3} y^{3}+\mathcal{A}_{2} y^{2}+\mathcal{A}_{1} y+\mathcal{A}_{0}=0$,

$\mathcal{D}_{3} \varepsilon^{3}+\mathcal{D}_{2} \varepsilon^{2}+\mathcal{D}_{1} \varepsilon+\mathcal{D}_{0}=0$

where the coefficients are given as

$\mathcal{A}_{4}=64 \pi^{3}\left(1+3 \lambda_{2}\right)$,

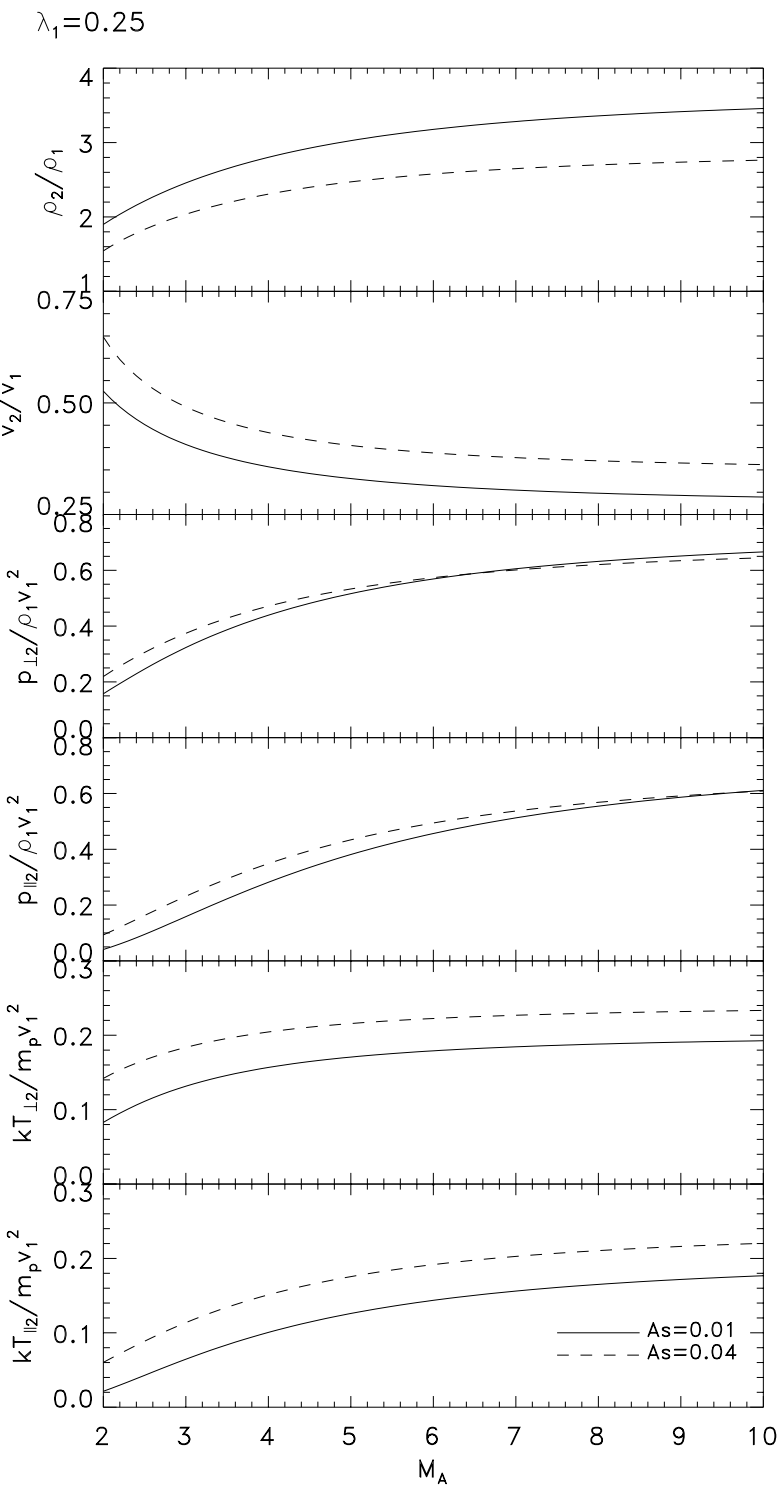

Fig. 4. Plasma parameters as functions of the Alfvén Mach number for the perpendicular shock for two different values of $A_{S}$.

$$
\begin{aligned}
\mathcal{A}_{3}= & 16 \pi^{3} B_{n 1}^{2}\left(1+2 \lambda_{2}\right)-64 \pi^{3} I_{1}\left(1+4 \lambda_{2}\right) \\
& -16 \pi^{2} B_{n 1}^{2} \varepsilon\left(3+8 \lambda_{2}\right), \\
\mathcal{A}_{2}= & 4 \pi B_{n 1}^{4} \varepsilon\left[\varepsilon\left(3+7 \lambda_{2}\right)-2\left(1+2 \lambda_{2}\right)\right] \\
& +32 \pi^{2} B_{n 1}^{2} I_{1} \varepsilon\left(1+4 \lambda_{2}\right)+64 \pi^{3} \lambda_{2}\left(2 W_{1}-J_{1}^{2}\right), \\
\mathcal{A}_{1}= & 8 \pi^{2}\left(H_{1}+B_{n 1} J_{1}\right)^{2}+B_{n 1}^{6} \varepsilon^{2}\left(1+2 \lambda_{2}\right)(1-\varepsilon) \\
& +32 \pi^{2} B_{n 1}^{2} \lambda_{2} \varepsilon\left(J_{1}^{2}-2 W_{1}\right)-4 \pi B_{n 1}^{4} I_{1} \varepsilon^{2}\left(1+4 \lambda_{2}\right), \\
\mathcal{A}_{0}= & 4 \pi B_{n 1}^{2} \lambda_{2} \varepsilon^{2}\left(2 B_{n 1}^{2} W_{1}+2 B_{n 1} J_{1} H_{1}+H_{1}^{2}\right),
\end{aligned}
$$

and

$$
\begin{aligned}
\mathcal{D}_{3}= & -B_{n 1}^{6}, \\
\mathcal{D}_{2}= & B_{n 1}^{4}\left[B_{n 1}^{2}+4 \pi y\left(3-\lambda_{2}\right)-4 \pi I_{1}\left(1-\lambda_{2}\right)\right], \\
\mathcal{D}_{1}= & 16 \pi^{2} B_{n 1}^{2} y^{2}\left(2 \lambda_{2}-3\right)+8 \pi B_{n 1}^{2} y\left[4 \pi I_{1}\left(1-\lambda_{2}\right)\right] \\
& -8 \pi B_{n 1}^{4} y-16 \pi^{2} \lambda_{2}\left(J_{1} B_{n 1}+H_{1}\right)^{2},
\end{aligned}
$$




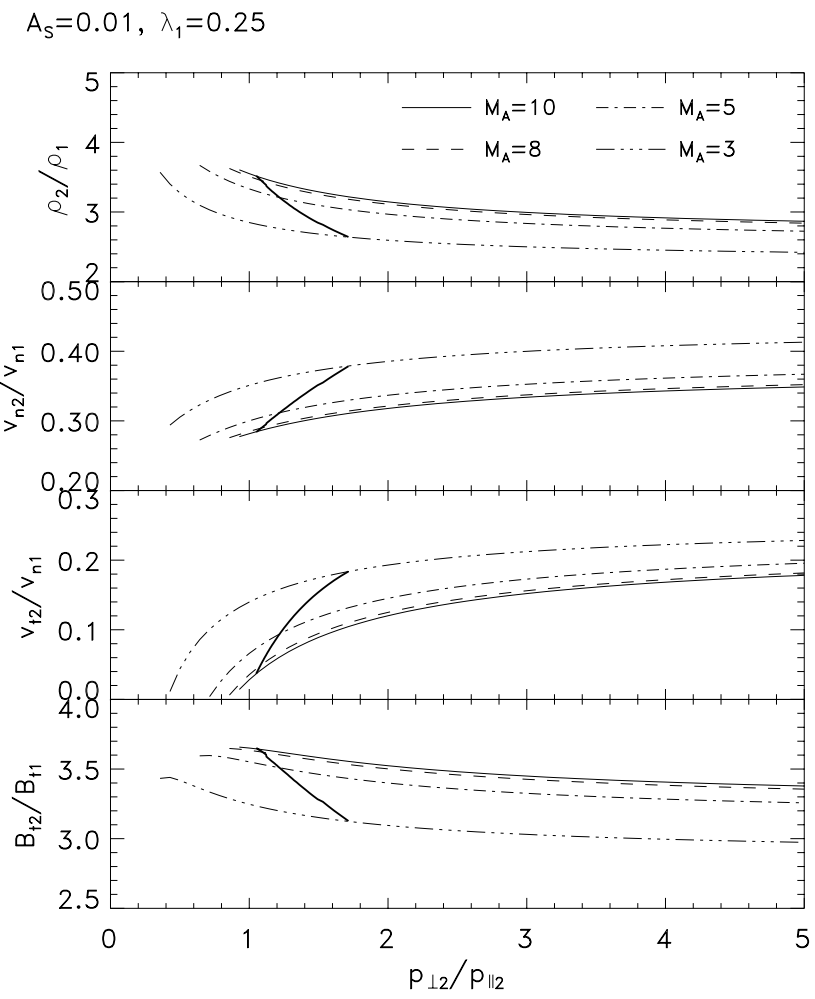

Fig. 5. Plasma parameters and magnetic field strength as functions of the anisotropy rate downstream of the oblique shock for $A_{S}=$ $0.01, \lambda_{1}=0.25$, and $\gamma=45^{\circ}$.

$$
\begin{aligned}
\mathcal{D}_{0}= & 64 \pi^{3} y^{2}\left(1-\lambda_{2}\right)\left(y-I_{1}\right)+16 \pi^{2} B_{n 1}^{2} y^{2} \\
& +8 \pi^{2}\left(1+\lambda_{2}\right)\left(J_{1} B_{n 1}+H_{1}\right)^{2} .
\end{aligned}
$$

Here, $H_{1}$ is the tangential component of the electric field, $I_{1}$ is the normal component of the momentum flux, $J_{1}$ is the tangential component of momentum flux, and $W_{1}$ is the energy flux upstream of the shock,

$$
\begin{aligned}
H_{1}= & \sqrt{4 \pi A_{M}} \sin \gamma, \\
I_{1}= & A_{S}+A_{S} \cos ^{2} \gamma\left(\frac{1}{\lambda_{1}}-1\right)+\frac{1}{2} A_{M} \sin ^{2} \gamma+1, \\
J_{1}= & \frac{1}{2} \sin (2 \gamma)\left[\frac{A_{S}}{\lambda_{1}}-A_{S}-A_{M}\right], \\
W_{1}= & A_{S}\left(2+\frac{1}{2 \lambda_{1}}+\frac{\cos ^{2} \gamma}{\lambda_{1}}-\cos ^{2} \gamma\right) \\
& +A_{M} \sin ^{2} \gamma+\frac{1}{2} .
\end{aligned}
$$

In Fig. 5 we show the variations of the plasma density, the normal and tangential components of the velocity and the tangential component of the magnetic field strength as functions of the pressure anisotropy downstream of the shock in the case of an oblique shock for different Alfvén Mach numbers, $M_{A}=3,5,8,10$ and a fixed parameter $A_{S}=0.01$. The angle between the normal vector of the discontinuity and the magnetic field upstream of the shock, $\gamma$, is chosen to be $45^{\circ}$. For the same shock, Fig. 6 shows the variations of the
$A_{S}=0.01, \lambda_{1}=0.25$

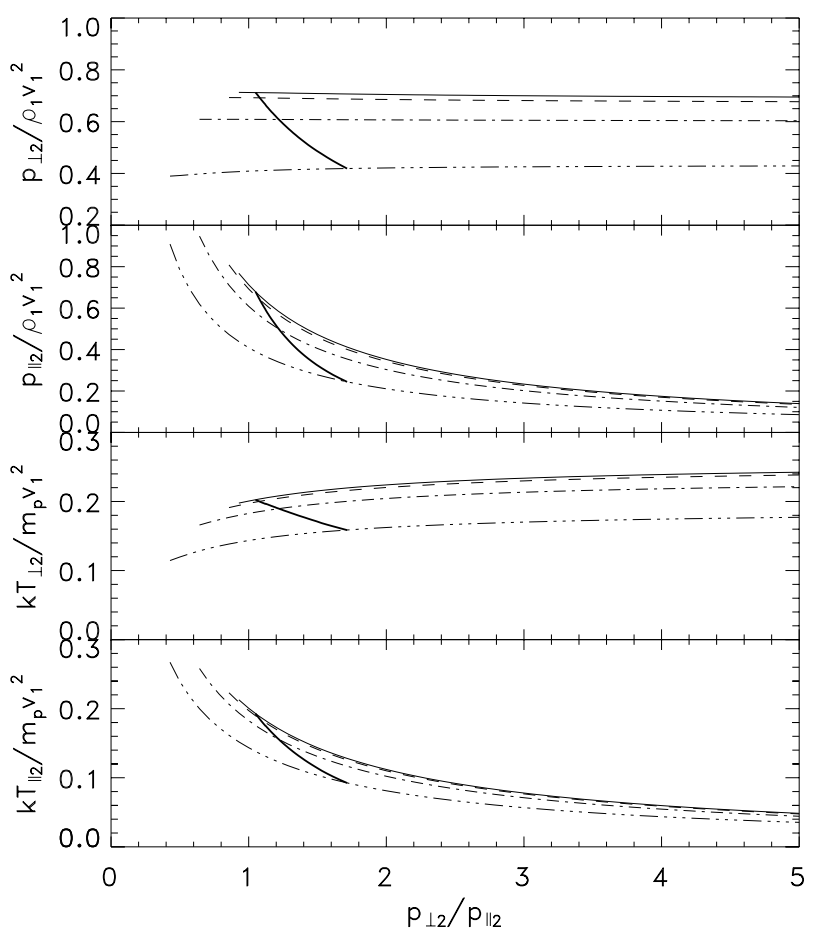

Fig. 6. Thermal plasma parameters as functions of the anisotropy rate downstream of the oblique shock for $A_{S}=0.01, \lambda_{1}=0.25$, and $\gamma=45^{\circ}$.

thermal quantities, i.e. the perpendicular and parallel pressures and temperatures, as functions of the anisotropy rate, $p_{\perp 2} / p_{\| 2}$. On the left side in each panel, all curves start from the points corresponding to the criterion of the fire-hose instability, $\beta_{\|}>2+\beta_{\perp}$. Similar to Figs. 2 and 3, the thick solid line appearing in each panel corresponds to the criterion of the mirror instability separating into stable (left side) and unstable (right side) regions. Figures 7 and 8 are similar to Figs. 5 and 6, but all calculations are done for $A_{S}=0.04$.

\subsubsection{Entropy across the shock}

In this paragraph, we concentrate on the change of the total entropy through the shock wave. For the thermodynamic reversible process, the entropy variation is related to the heat flux, $\delta Q$, and defined as $d S=(\delta Q) / T$, whereas for the irreversible thermodynamic process, the inequality $d S>(\delta Q) / T$ holds. In the case of pressure anisotropy, two degrees of freedom (perpendicular and parallel) are not in thermal equilibrium and hence, two adiabatic laws and two entropy functions are considered,

$S_{\perp}=k_{B} \ln \left(\frac{p_{\perp}}{\rho B}\right) \quad$ and $\quad S_{\|}=\frac{k_{B}}{2} \ln \left(\frac{p_{\|} B^{2}}{\rho^{3}}\right)$

where $k_{B}$ is the Boltzmann constant. Inside the shock front, the kinetic energy is converted into thermal energy and the 


$$
A_{s}=0.01, \lambda_{1}=0.25
$$

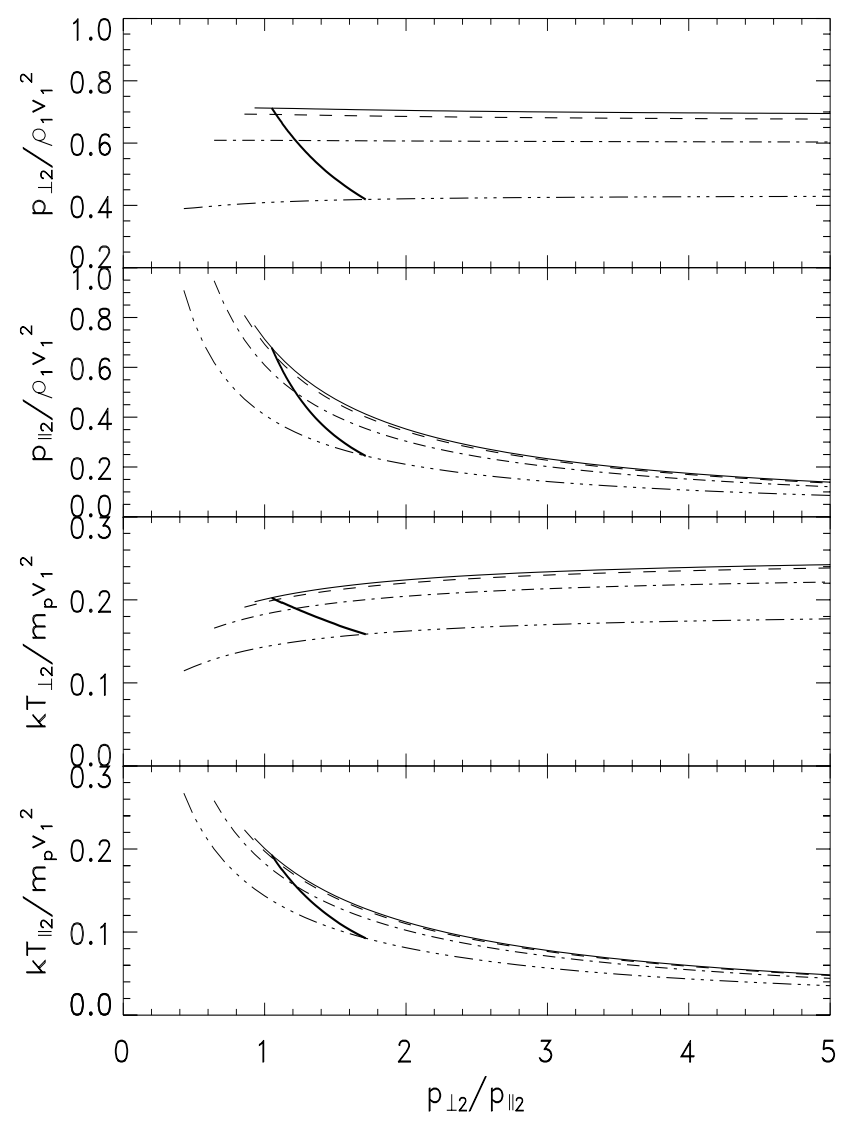

Fig. 7. Plasma parameters and magnetic field strength as functions of the anisotropy rate downstream of the oblique shock for $A_{S}=$ $0.04, \lambda_{1}=0.25$, and $\gamma=45^{\circ}$.

variations of the entropy functions are caused by heat fluxes,

$T_{\perp} d S_{\perp}=-q+q_{2} \quad$ and $\quad T_{\|} d S_{\|}=q+q_{1}$.

Here, $q_{1}$ and $q_{2}$ are the positive external heat fluxes, and $q$ is the exchange heat flux between perpendicular and parallel degrees of freedom. Summing up the Eqs. (27), one obtains the differential of the total entropy, $d S$,

$d S=\frac{q\left(T_{\perp}-T_{\|}\right)}{T_{\perp} T_{\|}}+\frac{q_{1}}{T_{\|}}+\frac{q_{2}}{T_{\perp}}>0$,

where

$S=S_{\perp}+S_{\|}=\frac{k_{B}}{2} \ln \left(\frac{p_{\perp}^{2} p_{\|}}{\rho^{5}}\right)$.

In the right side of Eq. (28), the second term is obviously positive because it is proportional to the heat flux related to the dissipation of kinetic energy of the plasma flow. The first term is proportional to the heat flux, which goes from the perpendicular to the parallel energy. From thermodynamics, it follows that this heat flux must be positive when $T_{\perp}>T_{\|}$, and negative, when $T_{\perp}<T_{\|}$. Therefore, in all cases, the first term in Eq. (28) must be positive, and hence, the variation

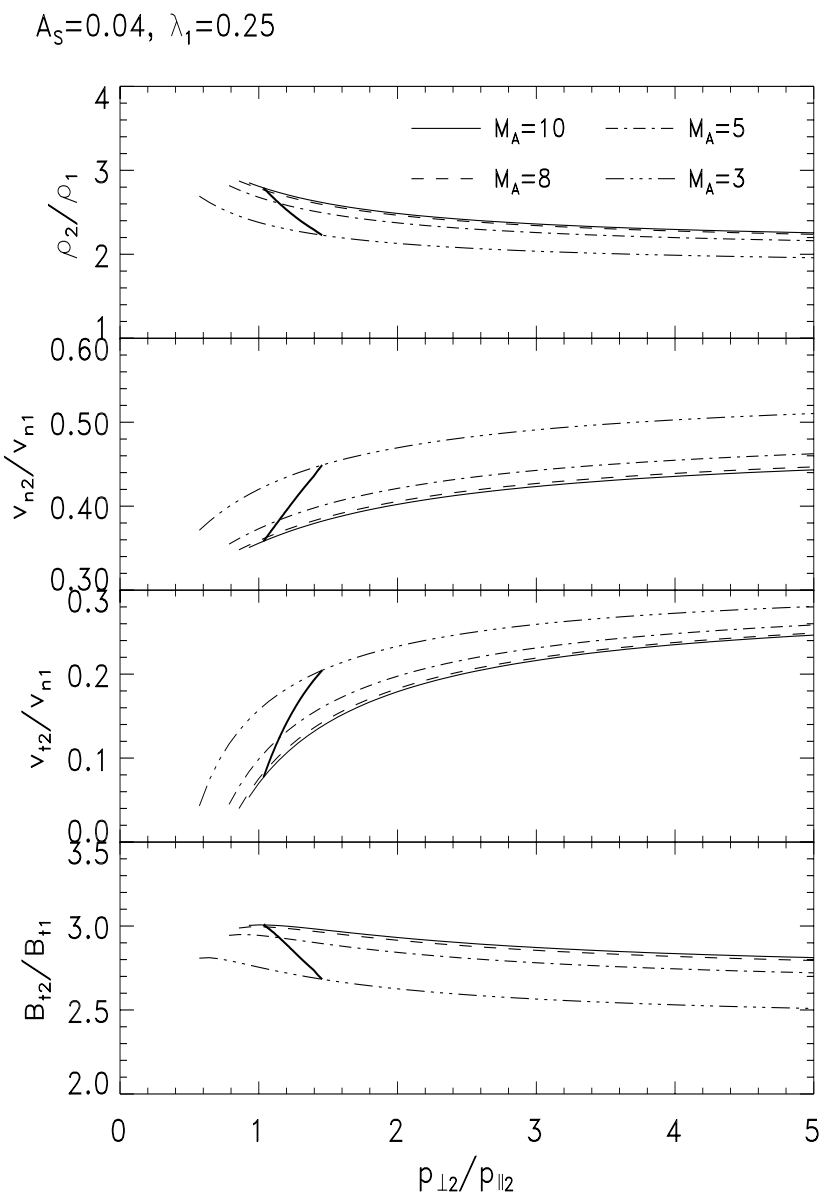

Fig. 8. Thermal plasma parameters as functions of the anisotropy rate downstream of the oblique shock for $A_{S}=0.04, \lambda_{1}=0.25$, and $\gamma=45^{\circ}$.

of the total entropy through the shock front must be positive. The increase of total entropy is due to two sources: a) dissipation processes in plasma and b) energy exchange between the parallel and perpendicular degrees of freedom. In the limit case of isotropy, the total entropy defined by Eq. (29) becomes equal to the usual entropy expression for the ideal gas with the polytropic index 5/3.

Figure 9 shows the total entropy difference at the fast shock as function of the pressure anisotropy for different Alfvén Mach numbers $\left(M_{A}=3,5,8,10\right)$, as obtained from our calculations. Panel a) and b) correspond to different parameters, i.e. $A_{S}=0.01$, and $A_{S}=0.04$. As seen from the figure, all curves indicate the positive difference of the total entropy, as required by (29).

\section{Comparison with a data example}

For comparing our theory with spacecraft data, we use plasma and magnetic field observations made by the WIND spacecraft for the time period from 19:00 UT until 20:00 UT on 30 November 1994. The data are shown as the dots in Fig. 10. They are measured during a subsolar pass of the 
a) $A_{s}=0.01, \lambda_{1}=0.25$

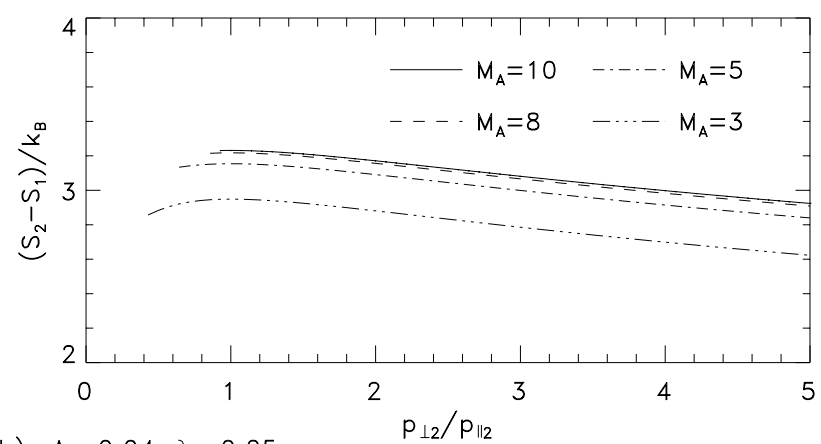

b) $A_{s}=0.04, \lambda_{1}=0.25$

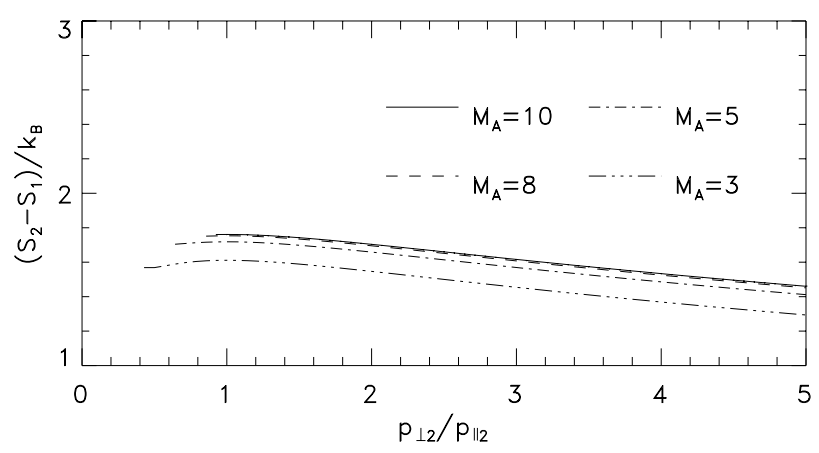

Fig. 9. Total entropy difference as function of the anisotropy rate downstream of the shock for different Alfvén Mach numbers: a) corresponds to $A_{S}=0.01$ and b) corresponds to $A_{S}=0.04$.

Earth's magnetosheath. Figure 10 shows, from top to bottom, the plasma density, the velocity, the magnetic field strength and the perpendicular and parallel pressures and temperatures with respect to the magnetic field. As seen from the figure, WIND crossed the Earth's bow shock at approximately 19:20 UT.

From the solar wind measurements (time period from 19:00 UT until 19:20 UT), we obtain the following input parameters for our theoretical analysis:

- normal vector: $n_{x}=0.99, n_{y}=0.10, n_{z}=-0.04$;

- dimensionless parameters: $A_{S}=0.0055, A_{M}=0.017$, and $\lambda_{1}=0.25$;

- angles: $\alpha=9.47^{\circ}$ and $\gamma=53.44^{\circ}$.

where the coplanarity theorem of the velocity

$\hat{\boldsymbol{n}}=\frac{\boldsymbol{v}_{2}-\boldsymbol{v}_{1}}{\left|\boldsymbol{v}_{2}-\boldsymbol{v}_{1}\right|}$

is used to calculate the normal vector of the discontinuity (Abraham-Shrauner and Yun, 1976). We note that these input parameters represent a four point averaging of the solar wind data close to the shock.

As seen from the figure, the shock shows an overshoot region, where the plasma density and the magnetic field strength have bigger values than the following data points (on average). In obtaining the input parameters using the

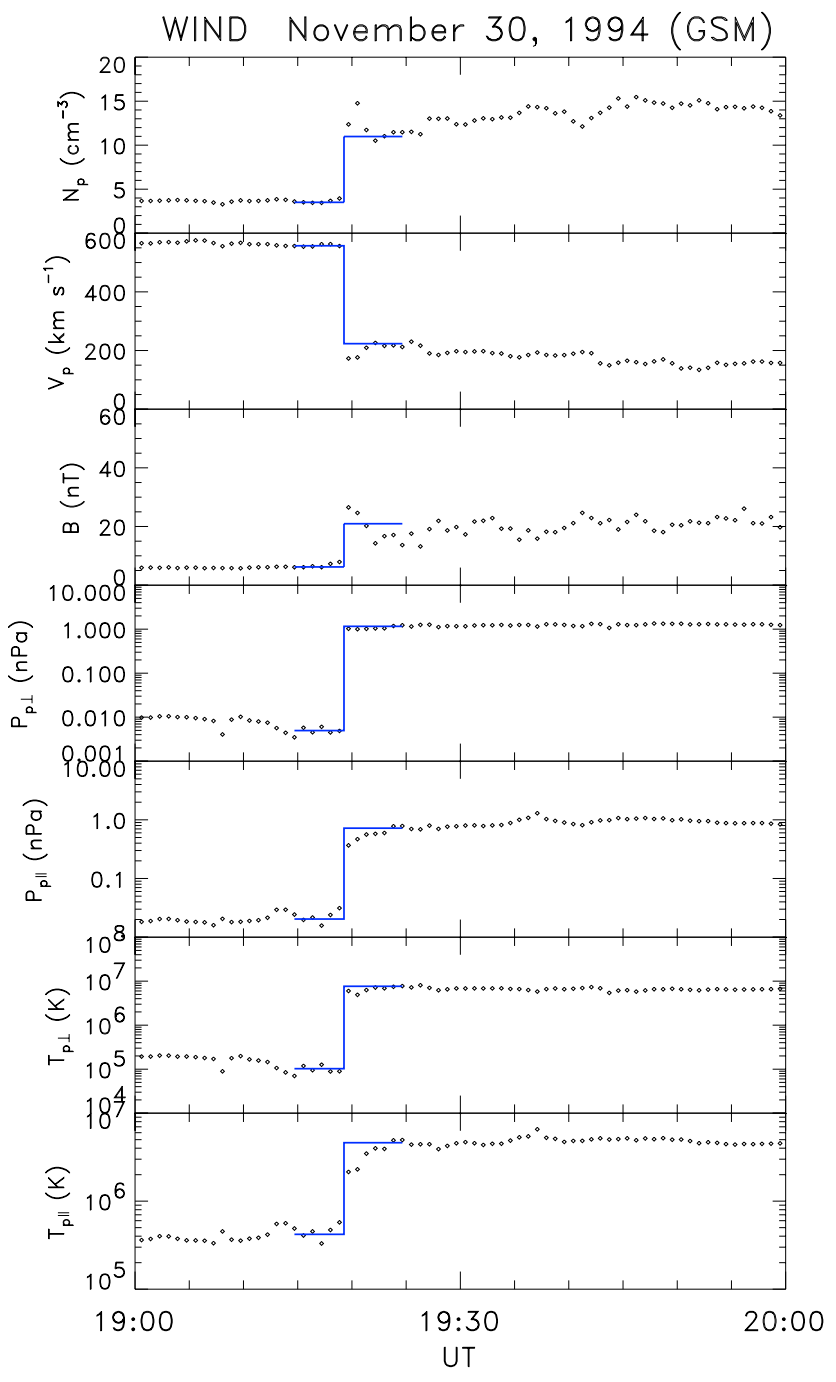

Fig. 10. Comparison of theoretical results with plasma parameters and magnetic field strength made by WIND spacecraft on 30 November 1994.

experimental data set, we do not take into account this overshoot region and consider only the points after it. As a next step, we use these input parameters to calculate the variations of the plasma parameters and magnetic field strength from upstream to downstream, as functions of the pressure anisotropy rate downstream of the shock. Then, we use the spacecraft observations to obtain the pressure anisotropy downstream of the shock, which is $\lambda_{2}=1.65$. Finally, we calculate the variations of the physical quantities across the shock with the above mentioned input parameters with the specific value of $\lambda_{2}$. The thick line appearing in each panel corresponds to our theoretical calculations. As seen from the figure, our theory fits the spacecraft data well; the averaged density jump from the data analysis is about $\rho_{2} / \rho_{1} \cong 3.17$, whereas our theory gives $\rho_{2} / \rho_{1} \cong 3.13$. 


\section{Discussion and conclusion}

We have reported on the analysis of the perpendicular and oblique fast shock for arbitrary pressure anisotropy upstream and downstream of the discontinuity. For solving the jump equations for an anisotropic magnetoplasma, the criteria of the mirror and fire-hose instability have been taken into account as additional equations and restrictions to the magnetoplasma on both sides of the shock. We analyzed all plasma parameters and the magnetic field strength downstream of the shock as functions of the anisotropy rate for different dimensionless parameters, $A_{M}$ and $A_{S}$, and a given pressure anisotropy rate upstream of the shock. Our results are useful for analyzing magnetosheath parameters for given solar wind conditions and are obtained without any assumptions regarding the Rankine Hugoniot jump equations.

For the perpendicular shock, the fire-hose instability plays no role as a bounding factor for low anisotropy rate. When the anisotropy parameter, $\lambda_{2}$, goes to zero, the density, parallel pressure, and parallel temperature strongly increase especially for high Alfvén Mach numbers.

Furthermore, we examined the solution for an oblique shock, which has an angle of $45^{\circ}$ between the magnetic field and the normal vector of the discontinuity. Some parameters have relatively small variations as functions of the anisotropy parameter $\lambda_{2}$. These parameters are density, normal component of the velocity, tangential component of the magnetic field, perpendicular pressure, and perpendicular temperature. Variations of other parameters, such as parallel pressure, parallel temperature, and the tangential component of the velocity are much more pronounced as functions of the anisotropy rate downstream of the shock. The total entropy was shown to increase through the fast shock front for a whole range of Alfvén Mach numbers.

In the last section, we compared experimental spacecraft data with our theory. The solar wind parameters were used as input parameters for our theoretical calculations. We further use this data set to obtain the pressure anisotropy downstream of the shock and calculate the variations of the plasma parameters, as well as the magnetic field strength for this specific value, which is $\lambda_{2}=1.65$. The difference between theory and observations turns out to be small, e.g. the averaged observed jump of the density is $\rho_{2} / \rho_{1} \cong 3.17$, whereas our theoretical calculations gives $\rho_{2} / \rho_{1} \cong 3.13$.

Acknowledgements. Part of this work was done while N. V. E. was on a research visit to the Space Research Institute of the Austrian Academy of Sciences in Graz and D. F. V. was on a research visit to the Institute for the Study of Earth, Oceans, and Space of the University of New Hampshire. This work is supported by the INTASESA Project 99-01277, by the Austrian "Fonds zur Förderung der wissenschaftlichen Forschung" grant P12761-TPH and grant P13804-TPH, by grant No 98-05-65290 from the Russian Foundation of Basic Research, by grant No 97-0-13.0-71 from the
Russian Ministry of Education, by NASA Grant NAG5-2834, and by the Austrian Academy of Sciences, "Verwaltungsstelle für Auslandsbeziehungen".

\section{References}

Abraham-Shrauner, B., Shock jump conditions for an anisotropic plasma, J. Plasma Phys., 1, 379, 1967.

Abraham-Shrauner, B. and Yun, S. H., Interplanetary shocks seen by Ames plasma probe on Pioneer 6 and 7, J. Geophys. Res., 81, 2097-2102, 1976.

Bame, S. J., Asbridge, J. R., Feldman, W. C., Gary, S. P., and Montgomery, M. D., Evidence for local ion heating in solar wind high speed streams, Geophys. Res. Lett., 2, 373, 1975.

Biernat, H. K., Erkaev, N. V., Farrugia, C. J., Vogl, D. F., Schaffenberger, W., and Kiendl, M. T., MHD solar wind flow around Earth, Jupiter, Saturn, and Venus: Similarities and peculiarities, Recent Research Developments in Plasmas, 1, 167-184, 2000.

Chao, J. K., Zhang, X. X., and Song, P., Derivation of temperature anisotropy from shock jump relations: Theory and observations, Geophys. Res. Lett., 22, 2409-2412, 1995.

Chew, G. F., Goldberger, M. L., and Low, F. E., The Boltzmann equation and the one-fluid hydromagnetic equations in the absence of particle collisions, Proc. Roy. Soc., A236, 112, 1956.

Erkaev, N. V., Vogl, D. F., and Biernat, H. K., Solution for jump conditions at fast shocks in an anisotropic magnetized plasma, J. Plasma Phys., in press, 2000.

Hasegawa, A., Plasma instabilities and nonlinear effects, Physics and Chemistry in Space, 8, Springer, Berlin, Heidelberg, New York, 1975.

Hill, P., Paschmann, G., Treumann, R. A., Baumjohann, W., and Sckopke, N., Plasma and magnetic field behavior across the magnetosheath near local noon, J. Geophys. Res., 100, 9575-9583, 1995.

Hudson, P. D., Discontinuities in an anisotropic plasma and their identification in the solar wind, Planet. Space Sci., 18, 1611, 1970.

Kiendl, M. T., Vogl, D. F., Biernat, H. K., and Erkaev, N. V., Collisional and collisionless MHD in space plasma physics, Recent Research Developments in Plasmas, 1, 199-218, 2000.

Neubauer, F. M., Jump relations for shocks in an anisotropic magnetized plasma, Z. Physik, 237, 205, 1970.

Lynn, Y. M., Discontinuities in an anisotropic plasma, research note, Physics of Fluid, 10, 2278, 1967.

Phan, T. D., Larson, D. E., Lin, R. P., McFadden, J. P., Anderson, K. A., Carlson, C. W., Ergun, R. E., Ashford, S. M., McCarthy, M. P., Parks, P. K., Réme, H., Bosqued, J. M., D’Uston, C., Wenzel, K.-P., Sanderson, T. R., and Szabo, A. The subsolar magnetosheath and magnetopause for high solar wind ram pressure: WIND observations, Geophys. Res. Lett., 23, 1279-1282, 1996.

Scarf, F. L., Wolfe, J. H., and Silva, R. W., A plasma instability associated with thermal anisotropies in the solar wind, J. Geophys. Res., 72, 993, 1967.

Vogl, D. F., Erkaev, N. V., Biernat, H. K., Mühlbachler, S., and Farrugia, C. J., Jump conditions at fast shocks in an anisotropic magnetized plasma, Cospar conference, Warszawa, submitted, 2000. 